\title{
Charge Transport by Superexchange in Molecular Host-Guest Systems
}

\author{
Franz Symalla, ${ }^{1}$ Pascal Friederich, ${ }^{1}$ Andrea Massé, ${ }^{2}$ Velimir Meded, ${ }^{1}$ Reinder Coehoorn, ${ }^{2}$ \\ Peter Bobbert, ${ }^{2}$ and Wolfgang Wenzel ${ }^{1, *}$ \\ ${ }^{1}$ Institute of Nanotechnology, Karlsruhe Institute of Technology, Karlsruhe, \\ Hermann von Helmholtz-Platz, 1,76344 Eggenstein-Leopoldshafen, Germany \\ ${ }^{2}$ Department of Applied Physics and Institute for Complex Molecular systems, \\ Eindhoven University of Technology, P.O. Box 513, \\ 5600 MB Eindhoven, The Netherlands
}

(Received 7 June 2016; revised manuscript received 25 July 2016; published 29 December 2016)

\begin{abstract}
Charge transport in disordered organic semiconductors is generally described as a result of incoherent hopping between localized states. In this work, we focus on multicomponent emissive host-guest layers as used in organic light-emitting diodes (OLEDs), and show using multiscale $a b$ initio based modeling that charge transport can be significantly enhanced by the coherent process of molecular superexchange. Superexchange increases the rate of emitter-to-emitter hopping, in particular if the emitter molecules act as relatively deep trap states, and allows for percolation path formation in charge transport at low guest concentrations.
\end{abstract}

DOI: 10.1103/PhysRevLett.117.276803

In amorphous organic semiconductors, energetic disorder of the electronic states on different molecules and weak electronic coupling between the van der Waals bonded molecules lead to localization of the charge carriers. Charge transport in such materials is commonly described as a result of thermally assisted quantum-mechanical tunneling ("hopping") between states localized on individual molecules [1]. This picture has been employed successfully to develop models for the steady state and transient electronic and optoelectronic performance of organic electronic devices based on disordered thin films, such as organic photovoltaic (OPV) devices and organic light-emitting diodes (OLEDs) [2-4]. However, it has not been established to what extent this view of charge transport as an incoherent process is applicable to all types of disordered organic semiconductors. Studies of charge transport in onedimensional molecular systems comprising a higher energy "bridge" unit separating two lower energy units suggest that transport to a distant neighbor via the coherent process of "superexchange" can be an important alternative to incoherent hopping transport [5-7]. The carrier then resides only virtually on the intermediate bridge unit. Similar mechanisms have been discussed in the context of other systems, where disorder plays no role. For organic donoracceptor co-crystals it has been predicted that conduction and valence bands are formed by superexchange coupling between spatially separated acceptors (donors) bridged by the noncovalently linked donors (acceptors), leading to a mirror symmetry of conduction and valence bands with remarkably small effective masses of electrons and holes [8-10]. In this Letter, we demonstrate that coherent superexchange processes can also contribute significantly to charge carrier mobility of disordered organic semiconductors. In view of the percolative nature of the three-dimensional transport process in such materials, the possible role of superexchange is not a priori evident. We study materials used in the emissive layer (EML) of phosphorescent OLEDs, and show that including transport via superexchange is highly relevant to quantitative analyses of their performance. The EML of state-of-the-art OLEDs consists of a mixture of a small concentration (typically 3-10 mol \%) of phosphorescent emissive dye molecules (guests), embedded in a host matrix of a charge transport material. Advantageously, the guest molecules act as exciton trapping sites, so that concentration quenching due to exciton diffusion is limited [11]. Furthermore, they function often also as charge trapping sites, allowing engineering of optimally shaped emission profiles [12]. The charge carriers reside then predominantly on the guest molecules. Experimental studies [13] and percolation based transport models [14] reveal a strong suppression of the carrier mobility in such systems, with a deep minimum for the dye concentration at the crossover between the lowconcentration regime in which the guest states act as isolated trap sites and the high-concentration regime in which direct guest-guest hopping is the dominant charge transport mechanism. We find that molecular superexchange, which provides a second pathway for guest-guest transport, can give rise to a strongly enhanced mobility near and beyond the crossover concentration.

The effect of virtual transfer to a host-type bridge molecule $B$ on the charge transfer rate between two weakly coupled guest-type molecules $A$ and $C$ may be evaluated as follows [see Fig. 1(a)]. Within the harmonic approximation of Marcus theory [15], the energies of the system with a charge on molecules $A, B$, or $C$ and with a relative molecular distortion (measured with respect to the neutral molecules) $x_{A}$ on $A$ and $x_{C}$ on $C$ are given by 


$$
\begin{gathered}
E_{A}\left(x_{A}, x_{C}\right)=E_{A, 0}+\frac{\lambda}{2}\left[\left(1-x_{A}\right)^{2}+x_{C}^{2}\right], \\
E_{B}\left(x_{A}, x_{C}\right)=E_{B, 0}+\frac{\lambda}{2}\left(x_{A}^{2}+x_{C}^{2}\right)+\frac{\lambda_{B}}{2},
\end{gathered}
$$

and

$$
E_{C}\left(x_{A}, x_{C}\right)=E_{C, 0}+\frac{\lambda}{2}\left[x_{A}^{2}+\left(1-x_{C}\right)^{2}\right]
$$

where $\lambda$ and $\lambda_{B}$ are the reorganization energy of the guest molecules $A$ and $C$, and host molecules $B$, respectively. $E_{A, 0}, E_{B, 0}$, and $E_{C, 0}$ are the energies of the fully relaxed states with a hole on molecules $A, B$, and $C$, respectively. Charge transfer from molecule $A$ to $C$ is viewed as a thermally activated process with an activation energy $E_{T}-E_{A, 0}$. From Marcus theory, the transition state energy

$$
E_{T}=E_{A, 0}+\frac{\left(E_{C, 0}-E_{A, 0}+\lambda\right)^{2}}{4 \lambda},
$$

is the energy for the distortion configuration at which the two parabolas defined by Eqs. 1(a) and 1(c) cross, i.e., for $x_{C}=1-x_{A}=\left(E_{C, 0}-E_{A, 0}+\lambda\right) /(2 \lambda) \equiv x_{T}$. The charge transfer rate is in Marcus theory then given by

$$
k_{A C}=\frac{2 \pi}{\hbar}\left|H_{A C}\right|^{2} \frac{1}{\sqrt{4 \pi \lambda k_{B} T}} \exp \left(-\frac{E_{T}-E_{A, 0}}{k_{B} T}\right),
$$

with $H_{A C}$ the transfer integral, $\hbar$ the reduced Planck constant, $k_{B}$ the Boltzmann constant, and $T$ the temperature. We extend this result by expressing the transfer integral as a sum of the direct electronic coupling and the superexchange coupling via bridge molecules $B_{i}$ (with $1 \leq i \leq N)$, using first-order perturbation theory:

$$
H_{A C} \cong H_{A C, 0}+\sum_{i=1}^{N} \frac{H_{A B_{i} 0} H_{B_{i} C, 0}}{\Delta E_{A B_{i} C}}
$$

with $H_{X Y, 0}$ the direct transfer integrals between molecules $X$ and $Y$ and with

$$
\begin{aligned}
\Delta E_{A B C} & =E_{\text {virtual }}-E_{T}=E_{B}\left(1-x_{T}, x_{T}\right)-E_{T} \\
& =E_{B, 0}-\frac{E_{A, 0}+E_{C, 0}}{2}+\frac{\lambda_{B}}{2} .
\end{aligned}
$$

The energy of the system in its virtual state with the charge on molecule $B$ and in the distortion configuration of the transition state $E_{\text {virtual }}$ has been deduced using Eq. 1(b). The last term in this symmetric expression is consistent with the notion that the bridge molecule does not respond to its occupation by relaxation to its minimum energy configuration, as its occupation is only virtual.
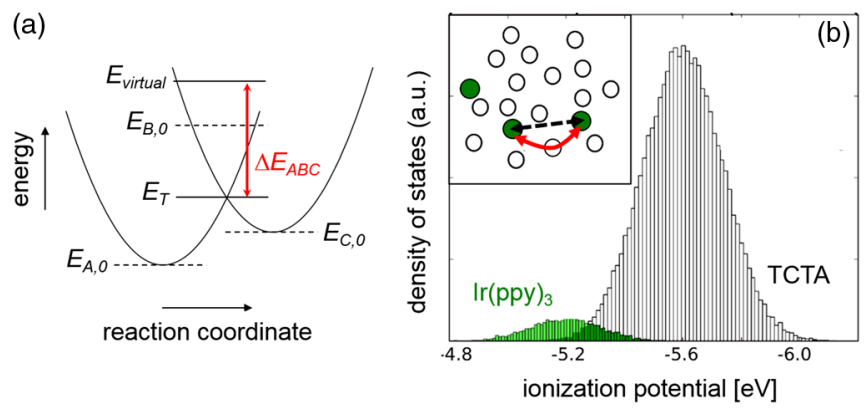

FIG. 1. (a) Energy level diagram of a guest (A)-host (B)-guest $(C)$ molecular subsystem, as defined in the main text, indicating the transition state energy $E_{T}$ and the virtual state energy $E_{\text {virtual, }}$ which determine the energy denominator $\Delta E_{\mathrm{ABC}}$ in the expression for the superexchange contribution to the charge transfer from molecule $A$ to $C$ [Eq. (5)]. (b) Calculated hole density of states for the case of $8 \mathrm{~mol} \% \operatorname{Ir}(p p y)_{3}$ (green, width $\sigma=0.118 \mathrm{eV}$ ) in a TCTA matrix (white, $\sigma=0.136 \mathrm{eV}$ ). The inset illustrates the spatial separation of the $\operatorname{Ir}(p p y)_{3}$ molecules in the matrix. Hole transport between the $\operatorname{Ir}(p p y)_{3}$ molecules is the combined result of direct (dashed arrow) and superexchange (full red arrow) interactions.

We quantify the hole transport in two prototypical host-guest systems frequently used in OLEDs: $\alpha$-NPD: $\operatorname{Ir}(\mathrm{MDQ})_{2}, \quad\left[N, N^{\prime} \mathrm{Di}(1\right.$-naphthyl)- $N$, $N^{\prime}$-diphenyl-(1, 1'-biphenyl)-4, 4'-diamine doped with the red emitting dye bis(2-methyldibenzo $f, h]$ quinoxaline) (acetylacetonate) iridium(III)), and TCTA: $\operatorname{Ir}(p p y)_{3}$ [tris (4-carbazoyl-9-ylphenyl)amine] doped with the greenemitting dye tris[2-phenylpyridinato- $\mathrm{C} 2, N]$ iridium(III)] These materials are used as the red- and green-emitting layers in OLEDs (e.g., Ref. [16]). Atomistic morphologies were generated by means of a Monte Carlo protocol that simulates vapor deposition [17]. These morphologies are subsequently employed to calculate the molecular hole reorganization energies, hole energies (ionization potentials) and hole transfer integrals using the quantum patch method [18] (see also Supplemental Material [19], Sec. 2).

As a first step to model hole transport in $\alpha$-NPD: $\operatorname{Ir}(\mathrm{MDQ})_{2}(a c a c)$ and TCTA: $\operatorname{Ir}(p p y)_{3}$ we have generated atomistic morphologies for both systems containing 1000 molecules per morphology, with a guest concentration of $8 \mathrm{~mol} \%$. For both systems, we find a mass density of about $0.87 \mathrm{~g} / \mathrm{cm}^{3}$. The inset of Fig. 1(b) provides a schematic view of the positionally disordered molecular structure. Pair distribution functions are shown in the Supplemental Material [19] (Fig. S1). The density of states (DOS) of many single-component disordered materials can be well approximated by a single Gaussian distribution. However, in the case of host-guest systems we have a superposition of two Gaussians, as shown in Fig. 1(b) for $8 \mathrm{~mol} \% \operatorname{Ir}(p p y)_{3}$ in a TCTA matrix. The offset between the two Gaussians is adjusted to reproduce that of the host and guest ionization potentials of $0.3 \mathrm{eV}[0.4 \mathrm{eV}]$ for the $\alpha$-NPD: $\operatorname{Ir}(\mathrm{MDQ})_{2}($ acac $)$ 


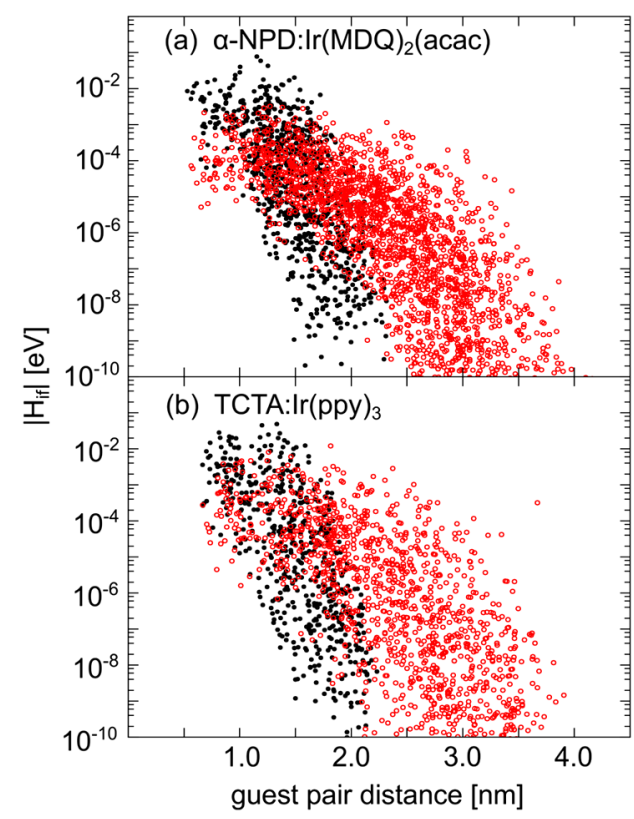

FIG. 2. Direct (black closed circles) and superexchange (red open circles) transfer integrals between guest molecules and adjacent molecules (guest and host) as a function of their center of mass distance for (a) $\operatorname{Ir}(\mathrm{MDQ})_{2}$ (acac) molecules in an $\alpha$-NPD host and (b) $\operatorname{Ir}(p p y)_{3}$ molecules in a TCTA host, for a guest concentration of $8 \mathrm{~mol} \%$.

[TCTA: $\left.\operatorname{Ir}(p p y)_{3}\right]$ system, as determined from ultraviolet photoelectron spectroscopy measurements [16]. The transfer integrals for direct and superexchange guest to guest/host hops are presented in Fig. 2. For small guest to guest/host distances, the direct transfer integrals are dominant. However, for distances above approximately $2 \mathrm{~nm}$ the superexchange transfer integrals are orders of magnitude larger than the direct transfer integrals.

Systems that can be treated realistically in explicit microscopic calculations are at present much smaller than those required to capture mesoscale effects like percolative charge transport [21]. We have therefore used a stochastic expansion method to generate mesoscale morphologies, with sizes from $67 \times 67 \times 67$ to $251 \times 125 \times 125 \mathrm{~nm}^{3}$. Expanded morphologies are stochastically generated to match the nearest-neighbor distance distributions of the atomistic morphology using a modified dominance competition model [22] (see Supplemental Material [19], Secs. 3-5). The on-site energies are distributed following the Gaussian distributions obtained from the atomistic morphologies and the transfer integrals are drawn from distance dependent probability distributions matching the microscopic calculations. Based on the data from this expansion, we study the importance of superexchange for the hole mobility of host-guest systems using kinetic Monte Carlo (KMC) simulations [23,24]. Simulation details are given in the Supplemental Material [19] (Sec. 6). Figure 3(a) shows for both systems the calculated guest concentration dependence of the hole mobility at various temperatures, without and including the superexchange transfer integrals. The hole concentration is $2.0 \times 10^{-4}$, a typical value in OLEDs, and the electric field is $0.03 \mathrm{~V} / \mathrm{nm}$. All curves show the characteristic mobility minimum at the crossover between the low concentration regime in which the guest states act as trap sites and the high-concentration regime in which the transport is due to direct guest-guest transport. The mobility reduction is strongly dependent on the material system, and is larger for the TCTA: $\operatorname{Ir}(p p y)_{3}$ system (0.4 eV average trap depth) than for the $\alpha$-NPD: $\operatorname{Ir}(\mathrm{MDQ})_{2}($ acac $)$ system (0.3 eV average trap depth). We find that superexchange increases the hole mobility at room temperature by up to a factor of 7 for TCTA: $\operatorname{Ir}(p p y)_{3}$ and a factor of 2 for $\alpha$-NPD: $\operatorname{Ir}(\mathrm{MDQ})_{2}($ acac $)$. With increasing temperature the effect decreases, which can be interpreted in terms of improved temperature-induced detrapping. Superexchange gives rise to enhanced guest-guest transport, so that the guest concentration at which the mobility minimum occurs becomes smaller. In particular for TCTA: $\operatorname{Ir}(p p y)_{3}$ the shift is significant, as may be seen from the temperature dependence of the concentration at which the minimum is found without and including superexchange, shown in Fig. 3(b). The lower value of $c_{\min }$ at which the minimum occurs and its increased temperature dependence can provide evidence for the importance of superexchange in experiments on a particular host-guest system. We note that an increase of the hole concentration to $1.0 \times 10^{-3}$ increases the mobility by up to 2 orders of magnitude for TCTA: $\operatorname{Ir}(p p y)_{3}$, but does not influence the mobility increase by superexchange (see Supplemental Material [19], Sec. 8).

In order to assess the general relevance of detrapping due to superexchange-mediated hops, we consider a model system consisting of a host molecule $B$ sandwiched between two guest molecules $A$ and $C$, in which a charge is trapped on molecule $A$. For simplicity, we assume the same reorganization energy $\lambda=0.2 \mathrm{eV}$ for all molecules, no direct coupling of molecules $A$ and $C\left(H_{A C, 0}=0\right)$, equal host-guest transfer integrals $\left(H_{A B, 0}=H_{B C, 0} \equiv H_{h g}\right)$, and resonant guest states $\left(E_{C, 0}=E_{A, 0}\right)$. The ratio of the escape rate $\omega_{A C, s x}$ due to a superexchange-mediated hop from $A$ to $C$ and the escape rate $\omega_{A B, 0}$ due to a direct hop to $B$ depends then on the trap depth $\Delta E_{t r} \equiv E_{B, 0}-E_{A, 0}$ as (see Supplemental Material [21], Sec. 9)

$$
\frac{\omega_{A C, s x}}{\omega_{A B, 0}}=\left(\frac{H_{h g}}{\Delta E_{t r}+\lambda / 2}\right)^{2} \exp \left(\frac{\left(2+\Delta E_{t r} / \lambda\right) \Delta E_{t r}}{4 k_{B} T}\right) .
$$

In Fig. 4(a) this detrapping rate ratio is given as a function of the host-guest transfer integral $H_{h g}$ and the trap depth $\Delta E_{t r}$. Taking $H_{h g} \cong 10^{-3} \mathrm{eV}$ as a typical relevant value (the value at the distance of about $2 \mathrm{~nm}$ above which from Fig. 2 superexchange is seen to play a dominant role), the figure indicates a significant increase of the escape rate 

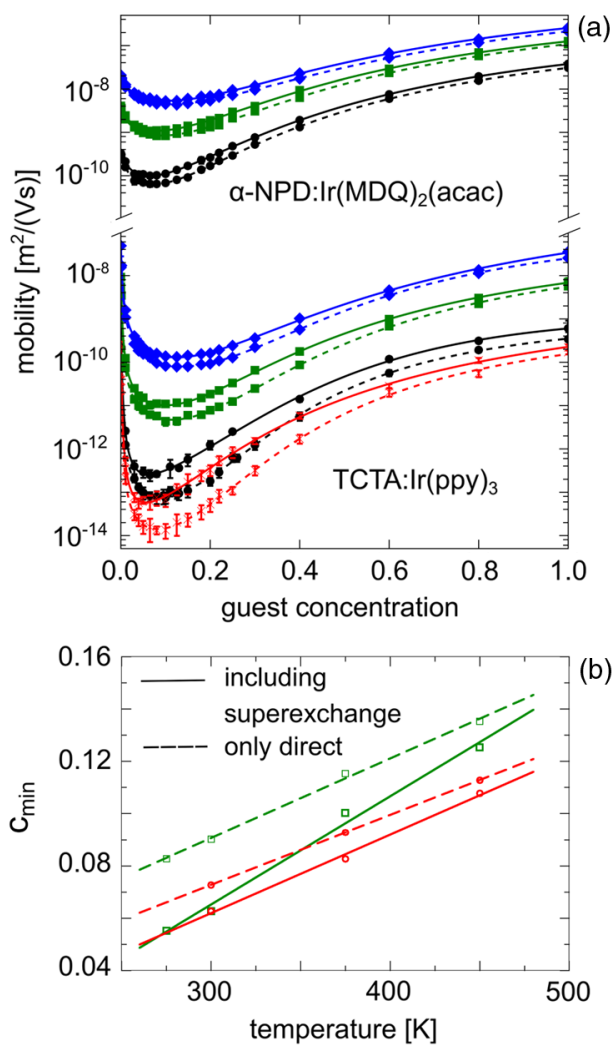

FIG. 3. (a) Guest concentration dependence of the hole mobility of $\alpha$-NPD $: \operatorname{Ir}(\mathrm{MDQ})_{2}(a c a c)$ and TCTA: $\operatorname{Ir}(p p y)_{3}$ systems at a hole concentration of $2.0 \times 10^{-4}$ per molecule and a field of $0.03 \mathrm{~V} / \mathrm{nm}$, at 275 (red crosses), 300 (black discs), 375 (green squares), and $450 \mathrm{~K}$ (blue diamonds). (b) Guest concentration at the mobility minimum, $c_{\min }$, as a function of the temperature. Red circles: $\alpha$-NPD: $\operatorname{Ir}(\mathrm{MDQ})_{2}($ acac $)$. Green squares: TCTA: $\operatorname{Ir}(p p y)_{3}$. The lines and curves in (a) and (b) are guides to the eye. Dashed lines: only direct hops. Solid lines: direct and superexchange hops.

by a superexchange-mediated hop for $\Delta E_{t r}=0.4 \mathrm{eV}$ [the average ionization potential difference for TCTA: $\left.\operatorname{Ir}(p p y)_{3}\right]$, and only a relatively small effect for $\Delta E_{t r}=0.3 \mathrm{eV}$, which is more representative for $\alpha$-NPD: $\operatorname{Ir}(\mathrm{MDQ})_{2}($ acac $)$. Qualitatively, this finding agrees well with the KMC simulation results. As molecules used in typical OLED materials are of similar size and as their frontier orbitals are generally $\pi$ orbitals of similar delocalization, the transfer integrals in most amorphous OLED materials are of a similar order of magnitude as for the two systems treated here. We thus conclude that superexchange-mediated hopping is likely to be the prevalent guest-guest transport mechanism for trap depths deeper than $0.3-0.4 \mathrm{eV}$.

Figure 4(a) reveals a strong dependence of the hopping rate enhancement by superexchange on the host-guest transfer integral. Off-diagonal disorder, i.e., a spread in transfer integral values, is, therefore, expected to have a strong influence on the nature of hole transport in guesthost systems and on the impact of superexchange. This is

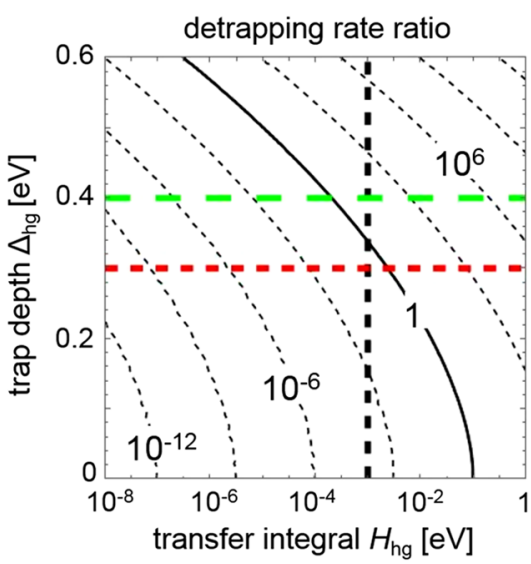

(a)

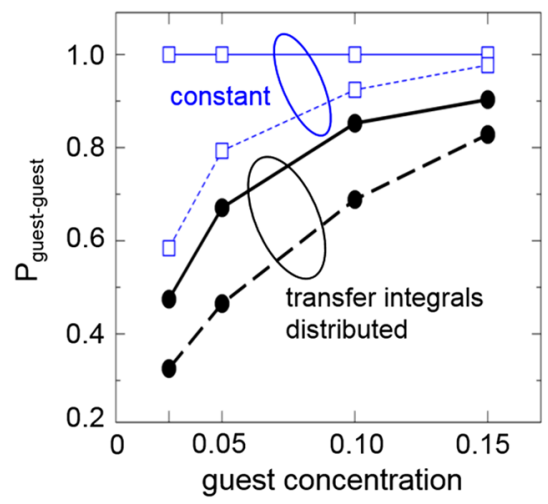

(b)

FIG. 4. (a) Contours of equal ratio between the superexchange hop rate from a trap $A$ to a resonant trap $C$ through a virtual state on host molecule $B$ and the hop rate from a trap $A$ to $B$, as a function of the host-guest transfer integral $H_{h g}$ and the trap depth $\Delta E_{t r}$. The red short-dashed and the green long-dashed horizontal lines at $\Delta E_{t r}=0.3(0.4) \mathrm{eV}$ indicate representative values for the $\alpha$-NPD: $\operatorname{Ir}(\mathrm{MDQ})_{2}(a c a c) /\left[\mathrm{TCTA}: \operatorname{Ir}(p p y)_{3}\right]$ system. The vertical dashed line indicates a representative value for $\mathrm{H}_{h g}$. (b) Guest concentration dependence of the probability of guest-guest hopping for equilibrated holes in TCTA: $\operatorname{Ir}(p p y)_{3}$ at $275 \mathrm{~K}$ with a hole concentration of $2.0 \times 10^{-4}$, in a model employing the calculated distribution of the transfer integrals (black discs) and using constant transfer integrals (blue squares). Dashed curves: only direct hops. Full curves: direct and superexchange hops.

confirmed by a further analysis of the simulation results. Figure 4(b) shows the guest concentration dependence of the average probability of guest-guest hopping for the equilibrium distribution of holes in TCTA: $\operatorname{Ir}(p p y)_{3}$, considering two different hopping models: one with a constant value of $H_{h g}$ and one with the transfer integrals drawn from the microscopic distribution, which covers a range of 10 orders of magnitude (see Fig. 2). Both models have the same mean value of $H_{h g}$ and use the energy dependence of the rate as in the Marcus model. In the absence of offdiagonal disorder, the inclusion of superexchange results in a strong increase of the guest-guest hopping probabilities, to essentially $100 \%$, even at low guest concentrations. However, in the presence of off-diagonal disorder the effect of including superexchange is more moderate. 
This difference results from the fact that the number of accessible host molecules rises much faster with the hopping radius than the number of accessible guest molecules. Since the hopping matrix elements to either guest or host molecules are distributed over many orders of magnitude, the probability for a strongly connected host molecule is larger in the model with off-diagonal disorder. We note that offdiagonal disorder is often not considered in mesoscopic transport models. In such models, the importance of superexchange would be overestimated considerably.

In summary, we have shown that superexchangemediated hops are crucial to describe charge transport in typical host-guest systems important for OLEDs. Using a multiscale $a b$ initio based model, we find that superexchange hops can, in a realistic case, increase the mobility by up to 1 order of magnitude. We also find that the mobility minimum can shift to considerably lower guest concentrations when compared to models that consider only direct hops. The relevance of superexchange crucially depends on the energy difference between the average onsite hole energy of guest and host molecules: it plays an increasingly important role in host-guest systems for which this energy difference is large. The quantitative impact of superexchange is strongly affected by the presence of offdiagonal disorder and therefore cannot be properly described with lattice models that neglect the distance dependence and distribution of the hopping matrix elements. This conclusion is essential for using simulation tools to support the selection of host and guest materials for OLEDs with optimally balanced electron and hole mobilities. We note that superexchange may play an important role in other types of systems. It may be relevant to explain transport in mixed-matrix type host materials in smallmolecule OLEDs [25] and in polymer OLEDs in which copolymerization of a matrix material with hole-transporting units acting as traps is employed [26]. The effect can also play an important role in exciton transport [27]: the emitter molecules of the systems investigated in this Letter have triplet energies that are approximately $0.3-0.4 \mathrm{eV}$ smaller than the host triplet energies. Exciton hopping from guest to host is therefore heavily suppressed, and a superexchange mechanism could contribute to enhanced Dextertype [28] guest-to-guest exciton transport. Superexchange may also explain the hole transport mechanism in low donor concentration organic photovoltaic devices (OPV), where some small molecule materials operate at donor concentration down to 5\% [29,30]. Superexchange mediated donor-donor hopping may explain efficient hole transport down to such low concentrations. The abrupt breakdown of the current below 5\% donor concentration would then correspond to the breakdown of hole percolation similar to the OLED materials investigated here (see Fig. 3).

This work is part of the Dutch-German project "Modeling of organic light-emitting diodes: From molecule to device" (MODEOLED) and of the Horizon $2020 \mathrm{EU}$ projects EXTMOS (Project No. 646176) and MOSTOPHOS (Project No. 646259). MODEOLED is funded on the Dutch side by the Dutch Technology Foundation STW, the applied science division of NWO, and the Technology Program of the Dutch Ministry of Economic Affairs (Project No. 12200). On the German side, it is funded by the "Deutsche Forschungsgemeinschaft" (DFG; Project No. WE1863/22-1). The computations were performed on the computational resource ForHLR Phase I, funded by the Ministry of Science, Research and the Arts Baden-Württemberg, and the "Deutsche Forschungsgemeinschaft."

*Corresponding author. wolfgang.wenzel@kit.edu

[1] V. Coropceanu, J. Cornil, D. A. da Silva Filho, Y. Olivier, R. Silbey, and J.-L. Brédas, Chem. Rev. 107, 926 (2007).

[2] R. G. E. Kimber, E. N. Wright, S. E. J. O'Kane, A. B. Walker, and J. C. Blakesley, Phys. Rev. B 86, 235206 (2012).

[3] R. Coehoorn and P. A. Bobbert, Phys. Status Solidi A 209, 2354 (2012).

[4] P. Kordt, J. M. van der Holst, M. Al Helwi, W. Kowalsky, F. May, A. Badinski, C. Lennartz, and D. Andrienko, Adv. Funct. Mater. 25, 1955 (2015).

[5] N. S. Hush, Coord. Chem. Rev. 64, 135 (1985).

[6] D. Segal, A. Nitzan, W. B. Davis, M. R. Wasielewski, and M. A. Ratner, J. Phys. Chem. B 104, 3817 (2000).

[7] C. Lambert, G. Nöll, and J. Schelter, Nat. Mater. 1, 69 (2002).

[8] J. Zhang et al., Adv. Mater. 24, 2603 (2012).

[9] L. Zhu, Y. Yi, Y. Li, E. G. Kim, V. Coropceanu, and J. L. Bredas, J. Am. Chem. Soc. 134, 2340 (2012).

[10] L. Zhu, Y. Yi, A. Fonari, N. S. Corbin, V. Coropceanu, and J.-L. Brédas, J. Phys. Chem. C 118, 14150 (2014).

[11] Y. Kawamura, J. Brooks, J. J. Brown, H. Sasabe, and C. Adachi, Phys. Rev. Lett. 96, 017404 (2006).

[12] H. van Eersel, P. A. Bobbert, R. A. J. Janssen, and R. Coehoorn, Appl. Phys. Lett. 105, 143303 (2014).

[13] P. M. Borsenberger, E. H. Magin, and S. A. Visser, Jpn. J. Appl. Phys. 37, 1945 (1998).

[14] Y. Y. Yimer, P. A. Bobbert, and R. Coehoorn, J. Phys. Condens. Matter 20, 335204 (2008).

[15] R. A. Marcus, J. Chem. Phys. 24, 966 (1956).

[16] M. Mesta, M. Carvelli, R. J. de Vries, H. van Eersel, J. J. van der Holst, M. Schober, M. Furno, B. Lüssem, K. Leo, P. Loebl, R. Coehoorn, and P. A. Bobbert, Nat. Mater. 12, 652 (2013).

[17] T. Neumann, D. Danilov, C. Lennartz, and W. Wenzel, J. Comput. Chem. 34, 2716 (2013).

[18] P. Friederich, F. Symalla, V. Meded, T. Neumann, and W. Wenzel, J. Chem. Theory Comput. 10, 3720 (2014).

[19] See Supplemental Material at http://link.aps.org/ supplemental/10.1103/PhysRevLett.117.276803, which includes Ref. [20] for pair distribution functions and density of states of the materials, mobility dependence on charge carrier density as well as a discussion of the stochastic expansion method for morphology and transfer integrals, 
the kinetic Monte Carlo algorithm, and the ratio of superexchange and direct escape rates.

[20] V. Stehr, J. Pfister, R. Fink, B. Engels, and C. Deibel, Phys. Rev. B 83, 155208 (2011).

[21] A. Massé, R. Coehoorn, and P. A. Bobbert, Phys. Rev. Lett. 113, 116604 (2014).

[22] B. Baumeier, O. Stenzel, C. Poelking, D. Andrienko, and V. Schmidt, Phys. Rev. B 86, 184202 (2012).

[23] J. J. M. van der Holst, F. W. A. van Oost, R. Coehoorn, and P. A. Bobbert, Phys. Rev. B 83, 085206 (2011).

[24] V. Rodin, F. Symalla, V. Meded, P. Friederich, D. Danilov, A. Poschlad, G. Nelles, F. von Wrochem, and W. Wenzel, Phys. Rev. B 91, 155203 (2015).
[25] A. B. Chwang, R. C. Kwong, and J. J. Brown, Appl. Phys. Lett. 80, 725 (2002).

[26] S. L. M. van Mensfoort, J. Billen, M. Carvelli, S. I. E. Vulto, R. A. J. Janssen, and R. Coehoorn, J. Appl. Phys. 109, 064502 (2011).

[27] G. Scholes, Annu. Rev. Phys. Chem. 54, 57 (2003).

[28] D. L. Dexter, J. Chem. Phys. 21, 836 (1953).

[29] M. Zhang, H. Wang, H. Tian, Y. Geng, and C. W. Tang, Adv. Mater. 23, 4960 (2011).

[30] K. Vandewal, J. Widmer, T. Heumueller, C. J. Brabec, M. D. McGehee, K. Leo, M. Riede, and A. Salleo, Adv. Mater. 26, 3839 (2014). 